\title{
Higher Bruhat and Tamari Orders and their Realizations
}

\section{Dimakis $A^{1}$ and Müller-Hoissen $F^{2 *}$}

${ }^{1}$ Department of Financial and Management Engineering, University of the Aegean, Greece

${ }^{2}$ Max Planck Institute for Dynamics and Self-Organization, 37077 Göttingen, Germany

\begin{abstract}
We briefly describe recent results about higher Bruhat and Tamari orders, the associated simplex equations and generalizations (polygon equations) of the pentagon equation, and the appearance of these orders in soliton interactions.
\end{abstract}

Keywords: Associahedron; Higher Bruhat order; KP equation; Pentagon equation; Permutahedron; Simplex equation; Soliton; Tamari order; Yang-Baxter equation

\section{Introduction}

In this letter, we sketch some of our recent results [1] at the interface between integrable systems and combinatorics. This includes a revision of the relation between higher Bruhat orders and simplex equations [2,3], a decomposition of higher Bruhat orders, the resulting Tamari orders (expected to be equivalent to higher Stasheff -Tamari orders), and a new family of equations associated with the latter. We finally recall the occurrence of higher Bruhat and Tamari orders in a "tropical limit" of solitons of the famous Kadomtsev-Petviashvili (KP) equation $[4,5]$.

\section{Higher Bruhat Orders and Simplex Equations}

Let $[N]=\{1,2, \ldots, N\}$ and $\left(\begin{array}{c}{[N]} \\ n\end{array}\right)$ be the set of $n$-element subsets of $[N]$. A linear order (permutation) of $\left(\begin{array}{c}{[N]} \\ n\end{array}\right)$ is called admissible if for any $K \in\left(\begin{array}{c}{[N]} \\ n+1\end{array}\right)$, the packet $P(K):=\{n$-element subsets of $K\}$ is contained in it in lexicographical $(\vec{P}(K))$ or in reverse lexicographical order $(\bar{P}(K))$. Let $A(N, n)$ be the set of admissible linear orders of $\left(\begin{array}{c}{[N]} \\ n\end{array}\right)$. An equivalence relation is defined on $A(N, n)$ by setting $\rho$ $\sim \rho^{\prime}$ if $\rho$ and $\rho^{\prime}$ only differ by exchange of two neighboring elements, not both contained in some packet. The higher Bruhat order $B(N, n)$ is $A(N, n) / \sim$ supplied with the partial order determined by inversions $\vec{P}(K) \mapsto \bar{P}(K)$ of lexicographically ordered packets of neighboring elements. There is a natural correspondence between the elements of $A(N, n+1)$ and the maximal chains of $B(N, n)$. The Bruhat order $B(3,1)$ consists of the two maximal chains

$$
\begin{aligned}
& (1,2,3) \stackrel{12}{\rightarrow}(2,1,3) \stackrel{13}{\rightarrow}(2,3,1) \stackrel{23}{\rightarrow}(3,2,1) \\
& (1,2,3) \stackrel{23}{\rightarrow}(1,3,2) \stackrel{13}{\rightarrow}(3,1,2) \stackrel{12}{\rightarrow}(3,2,1)
\end{aligned}
$$

where $i j$ stands for $\{i, j\}$ and here indicates the inversion of the packet of it in the respective linear order. A set-theoretical realization

$$
i \mapsto \mathcal{U}_{i}, \quad(i, j, k) \mapsto \mathcal{U}_{i} \times \mathcal{U}_{j} \times \mathcal{U}_{k}, \quad i j \mapsto \mathcal{R}_{i j}: \mathcal{U}_{i} \times \mathcal{U}_{j} \rightarrow \mathcal{U}_{j} \times \mathcal{U}_{i}
$$

(or a realization using vector spaces and tensor products) leads to the Yang-Baxter (YB) equation

$$
\mathcal{R}_{23,12} \mathcal{R}_{13,23} \mathcal{R}_{12,12}=\mathcal{R}_{12,23} \mathcal{R}_{13,12} \mathcal{R}_{23,23}
$$

The boldface position indices are read off from the above chains. They specify on which pair of sets in the threefold direct product the map $\mathcal{R}_{i j}$ acts. In terms of $\hat{\mathcal{R}}_{i j}:=\mathcal{R}_{i j} \mathcal{P}$, where $\mathcal{P}$ is the transposition, the
YB equation has the more familiar form $\hat{\mathcal{R}}_{23,12} \hat{\mathcal{R}}_{13,13} \hat{\mathcal{R}}_{12,23}=\hat{\mathcal{R}}_{12,23} \hat{\mathcal{R}}_{13,13} \hat{\mathcal{R}}_{23,12}$.

For any $N>1$, the higher Bruhat order $B(N+1, N-1)$ consists of two maximal chains, so that it determines an equation in the same way as in the above example, where $N=2$. The associated equation is the $\mathrm{N}$-simplex equation. The 3 -simplex equation is thus a realization of $B(4,2)$. The two maximal chains of the latter poset are resolved below into elements of $A(4,2)$, and a linear order is now more conveniently displayed as a column. The coloring is referred to later on.

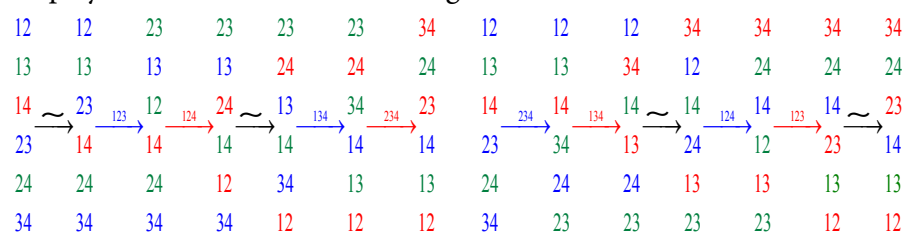

From the above chains we read off the 3 -simplex equation

$$
\mathcal{R}_{234,123} \mathcal{R}_{134,345} \mathcal{P}_{56} \mathcal{P}_{23} \mathcal{R}_{124,345} \mathcal{R}_{123,123} \mathcal{P}_{34}=\mathcal{P}_{34} \mathcal{R}_{123,456} \mathcal{R}_{124,234} \mathcal{P}_{45} \mathcal{P}_{12} \mathcal{R}_{134,234} \mathcal{R}_{234,456}
$$

for maps $\mathcal{R}_{i j k}: \mathcal{U}_{i j} \times \mathcal{U}_{i k} \times \mathcal{U}_{j k} \rightarrow \mathcal{U}_{j k} \times \mathcal{U}_{i k} \times \mathcal{U}_{i j}, i<j<k$. An equivalence $(\sim)$ is realized by a transposition map $\mathcal{P}_{a b}$ (acting at positions $\boldsymbol{a}$ and $\boldsymbol{b}$ ). In terms of $\hat{\mathcal{R}}_{i j k}:=\mathcal{R}_{i j k} \mathcal{P}_{13}: \mathcal{U}_{j k} \times \mathcal{U}_{i k} \times \mathcal{U}_{i j} \rightarrow \mathcal{U}_{j k} \times \mathcal{U}_{i k} \times \mathcal{U}_{i j}$ the 3 -simplex equation takes the form

$$
\hat{\mathcal{R}}_{1,123} \hat{\mathcal{R}}_{\hat{2}, 145} \hat{\mathcal{R}}_{3,246} \hat{\mathcal{R}}_{4,356}=\hat{\mathcal{R}}_{4,356} \hat{\mathcal{R}}_{\hat{3}, 246} \hat{\mathcal{R}}_{\hat{2}, 145} \hat{\mathcal{R}}_{1,123}
$$

using complementary notation: $\hat{1}=\{2,3,4\}=234$ etc. This is also known as tetrahedron equation, or Zamolodchikov equation. It appeared originally as a factorization condition for the S-matrix in a $(2+1)$-dimensional theory of "straight strings", and in a related three-dimensional exactly solvable lattice model (see, e.g., [6]).

The six linear orders constituting $A(3,1)$, viewed as maximal chains, build $B(3,0)$. It forms a cube, on which the structure of the YB equation is often visualized. There is a counterpart for any simplex equation. For example, the linear orders constituting $A(4,2)$ can be viewed as maximal chains of $B(4,1)$, which forms a polyhedron called

${ }^{*}$ Corresponding author: Müller-Hoissen F, Max Planck Institute for Dynamics and Self-Organization, 37077 Göttingen, Germany, Tel: 49551 5176-0; E-mail: Folkert. Mueller-Hoissen@ds.mpg.de

Received July 14, 2015; Accepted July 16, 2015; Published July 29, 2015

Citation: Dimakis A, Müller-Hoissen F (2015) Higher Bruhat and Tamari Orders and their Realizations. J Generalized Lie Theory Appl 9: e103. doi:10.4172/17364337.1000e103

Copyright: $\odot 2015$ Dimakis A, et al. This is an open-access article distributed under the terms of the Creative Commons Attribution License, which permits unrestricted use, distribution, and reproduction in any medium, provided the original author and source are credited. 


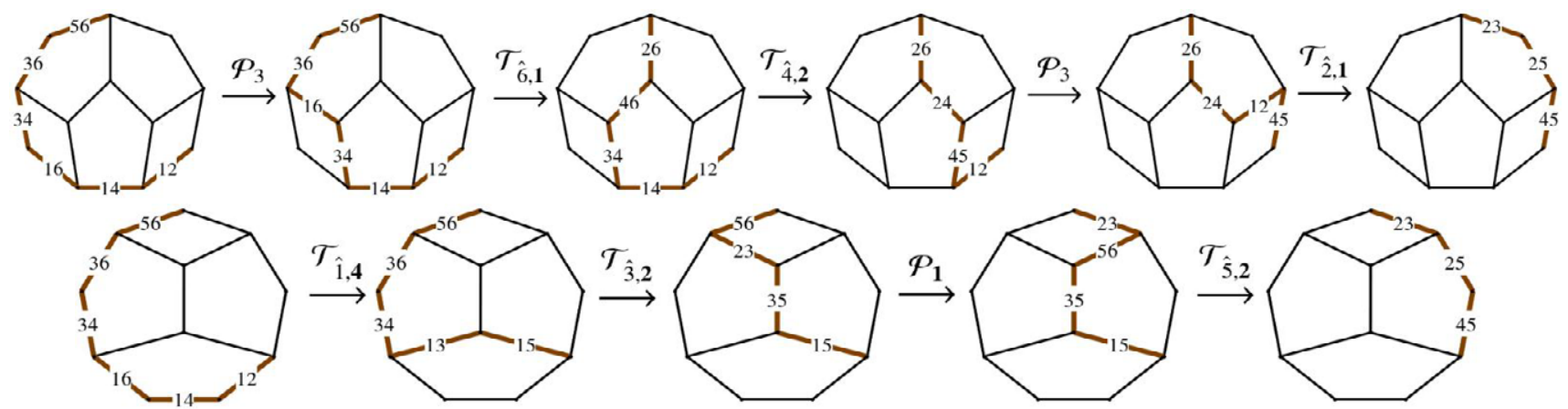

Figure 1: The structure of the left/right hand side of the hexagon equation on front/back side of the associahedron. Edge labels are in complementary notion (e.g., $\widehat{56}=1234$ ), but with hats omitted. We abbreviated consecutive position indices to the first $\left(e . g\right.$., $\left.\mathcal{T}_{\hat{i}, 4}=\mathcal{T}_{\hat{i}, 456}\right)$.

permutahedron, and the structure of the 3-simplex equation is nicely visualized on it [1].

$B(N+2, N-1)$ contains several copies of $B(N+1, N-1)$, and thus leads to a system of $N$-simplex equations. Let us "localize" them by allowing the maps to depend on additional variables, and such that each of these equations determines a map by taking the variables on the left hand side to those of the right hand side. Then, guided by $B(N+2$, $N)$, one can deduce that these maps have to satisfy the $(N+1)$-simplex equation [1]. Hence, the latter arises as the consistency condition of a system of localized $N$-simplex equations. This is a crucial integrability feature of the simplex equations.

\section{Decomposition of Bruhat Orders}

The above coloring of elements of the linear orders in the maximal chains of $B(4,2)$ indicates projections. The blue one results in

$$
(12,23,34) \stackrel{123}{\rightarrow}(13,34) \stackrel{134}{\rightarrow} 14 \quad(12,23,34) \stackrel{234}{\rightarrow}(12,24) \stackrel{124}{\rightarrow} 14
$$

and constitutes the Tamari order $T(4,2)$. The red projection of $B(4,2)$ is the dual of $T(4,2)$.

Crucial for the "decomposition" is the splitting $P(K)=P_{o}(K) \cup$ $P_{e}(K)$ of the packet of $K$ into the half-packets of elements with odd, respectively even position in the lexicographical order. The inversion of lexicographically ordered packets, which governs Bruhat orders, is replaced in a Tamari order by $\vec{P}_{o}(K) \mapsto \bar{P}_{e}(K)$. For the precise definitions we refer to [1].

\section{Polygon Equations}

The Tamari orders $T(N, N-2)$ consist of two maximal chains. We define the $N$-gon equation as a realization of it, now with maps $\mathcal{T}_{K}[1]$. For $N=5$, this is the ubiquitous pentagon equation. As for the YB equation, its structure can be visualized on a cube, which now carries $T(5,2)$, however. The hexagon equation realizes $T(6,4)$ and can be visualized on the associahedron (Stasheff polytope), formed by the Tamari lattice $T(6,3)$, see Figure 1.

The pentagon and hexagon equations appeared in realizations of Pachner moves of triangulations of a 3-, respectively 4-manifold [7,8]. The heptagon equation involves maps $\mathcal{T}_{i j k m p}: U_{i j k l m m} \times U_{i j k m p} \times U_{i k l m p} \rightarrow U_{j k k m p} \times U_{i j l m p} \times U_{i j k p}, i<j<k<l<m<p$. In terms of $\widehat{\mathcal{T}}_{K}:=\mathcal{T}_{K} \mathcal{P}_{13}$, it reads

$$
\widehat{\mathcal{T}}_{\hat{1}, 123} \widehat{\mathcal{T}}_{\hat{3}, 145} \widehat{\mathcal{T}}_{\hat{5}, 246} \widehat{\mathcal{T}}_{\hat{7}, 356}=\widehat{\mathcal{T}}_{\hat{6}, 356} \widehat{\mathcal{T}}_{\hat{4}, 245} \widehat{\mathcal{T}}_{\hat{2}, 123}
$$

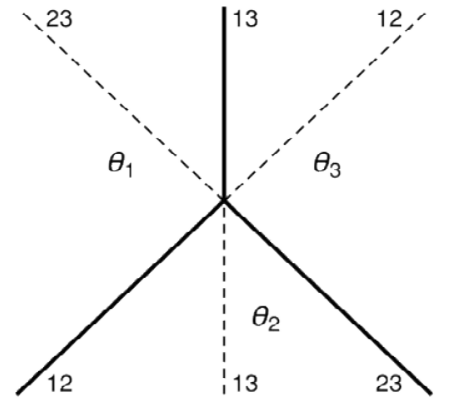

Figure 2: The solid half-lines form the boundaries between the three regions in the $x y$-plane, where $\max \left\{\theta_{1}, \theta_{2}, \theta_{3}\right\}$ equals one of $\theta_{i}, i=1,2,3$.

A related equation appeared in [9]. Apart from this, polygon equations are rather new terrain. Neighboring polygon equations are related by the same kind of "integrability" that connects neighboring simplex equations, and this is what makes them special and promising. The important role of the pentagon equation (some keywords are "multiplicative unitary", "Drinfeld associator", "quantum dilogarithm", see [1] for references) enhances the expectations for its higher analogs. The aforementioned decomposition of Bruhat orders leads to relations between (solutions of) simplex and polygon equations [1].

\section{Bruhat and Tamari Orders in Soliton Interactions}

Let $\theta_{i}:=p_{i} x+p_{i}^{2} y+p_{i}^{3} t+c_{i}$, with real constants $p_{i}, c_{i}, i=1,2, \ldots$, $m$. At fixed $\mathrm{t}, \theta_{i}=\theta_{i}$ determines the boundary line $i j$ between the region where $\theta_{i}>\theta_{j}$ and that where $\theta_{j}>\theta_{i}$. For $m=3$, there are three such lines, as shown in Figure 2. Proceeding from bottom to top ( $y$-direction), we observe the Bruhat order $B(3,2)$. Instead, the function $\max \left\{\theta_{1}, \ldots, \theta_{m}\right\}$ splits the $x y$-plane into regions, where one of the $\theta_{i}$ dominate, separated by a rooted and generically binary tree. For $m=3$, this is shown in Figure 2 (the dashed half-lines are then "invisible"). It realizes the Tamari order $T(3,2)$ and actually appears in nature as a "Miles resonance" on a fluid surface, mathematically described by a special soliton solution of the famous KP equation. Indeed, in a "tropical limit", where the above maximum function shows up, a subclass of KP soliton solutions realizes all the Tamari lattices $T(N, N-3)$ in terms of rooted binary trees [4,5]. Time evolution is then given by right rotation in a tree, which translates to the rightward application of the associativity law in Tamari's original presentation of the lattices (in terms of bracketings of a monomial of fixed length [10]). 
Citation: Dimakis A, Müller-Hoissen F (2015) Higher Bruhat and Tamari Orders and their Realizations. J Generalized Lie Theory Appl 9: e103. doi:10.4172/1736-4337.1000e103

Page 3 of 3

\section{Further Remarks}

Scattering of solitons leads to Yang-Baxter maps, i.e., settheoretical solutions of the YB equation (see e.g., [11]). Is there also a way to generate solutions of the simplex and polygon equations from (matrix) KP soliton interactions? For different aspects of KP soliton combinatorics, see, e.g., [12]. More generally, one can also ask for the combinatorics underlying soliton interactions in case of soliton equations other than KP. Surely, much more is there to be discovered.

\section{References}

1. Dimakis A, Müller-Hoissen F (2015) Simplex and polygon equations. SIGMA 11: 042 .

2. Manin $Y$, Schechtman $\vee$ (1986) Arrangements of real hyperplanes and Zamolodchikov equations. Group Theoretical Methods in Physics, eds. Markov M, Man'ko V, Dodonov V, VNU Science Press, Utrecht, The Netherlands, 151-165.

3. Manin Y, Schechtman V (1986) Higher Bruhat orders, related to the symmetric group. Funct Anal Appl 20: 148-150.

4. Dimakis A, Müller-Hoissen F (2011) KP line solitons and Tamari lattices. J Phys A Math Theor 44: 025203.
5. Dimakis A, Müller-Hoissen F (2012) KP solitons, higher Bruhat and Tamari orders. Associahedra, Tamari Lattices and Related Structures, vol. 299 of Progress in Mathematics, eds. Müller-Hoissen F, Pallo J, Stasheff J, (Birkhäuser, Basel) 391-423.

6. Stroganov $Y$ (1997) Tetrahedron equation and spin integrable models on a cubic lattice. Theor Math Phys 110: 141-167.

7. Korepanov I (2004) SL(2)-solution of the pentagon equation and invariants of three-dimensional manifolds. Theor Math Phys 138: 18-27.

8. Kashaev R (2015) On realizations of Pachner moves in 4D. Preprint arXiv:1504.01979 [math-ph].

9. Volkov A (1997) Beyond the 'pentagon identity'. Lett Math Phys 39: 393-397.

10. Tamari $D$ (1962) The algebra of bracketings and their enumeration. Nieuw Arch Wisk 10: 131-146.

11. Veselov A (2003) Yang-Baxter maps and integrable dynamics. Phys Lett A 314: $214-221$.

12. Kodama Y, Williams L (2014) KP solitons and total positivity for the Grassmannian. Invent math 198: 637-699. 